\title{
Financial Feasibility for Project Machining Under Bracket PT. Karya Komponen Presisi
}

\author{
Emmelia Tan' ${ }^{1}$, Fitria ${ }^{2}$ \\ Address: J1. Inspeksi Kalimalang Tegal Danas Arah Delta Mas Cikarang Selatan, Bekasi 17530, \\ Jawa Barat, Indonesia \\ emmelia.tan@pelitabangsa.ac.id ${ }^{1}$, fitriasyefrudin@gmail.com²
}

\begin{abstract}
The aumotive and machinary industry nowdays has been very competitive and volatile also. Automotive component producer should consider cautiously. This study do financial analysis of the new project 'Machining Under Bracket' in PT Karya Komponen Presisi. In addition, company wants to find minimum feasible selling price. Therefore, Capital Budgeting Methods are used for project feasibility analysis such as Payback Period (PP), Internal Rate of Returns (IRR), Net Present Value (NPV), and Profitability Index (PI), sensitivity analysis, risk analysis and decision tree. The research data are taken from previous similar project that has been done and used to forecast new project production cost, investment cost and sales forecast. The findings show the New Project „Machining Under Bracket“ feasible to run by the company with coefficient of variances is $19,97 \%$, minimum selling price IDR 20.349 /pcs, expected NPV IDR 1.227.613.848. In addition the result for normal condition IRR is $21,48 \%$, NPV as IDR 1.272.346.624, Payback Period will take three years and three months with Profitability index 1,157.
\end{abstract}

Keywords: Payback Period, Internal Rate of Returns, Net Present Value, Profitability Index, Financial Feasibility, Decision Tree, Risk Analysis, Sensitivity Analysis

\section{Introduction}

In 2016, United Nations Statistics Division, recorded Indonesia as the fourth ranking in manufacturing industry contribute to Gross Domestic Product after South Korea, China and German. In line with, Statistics Indonesia recorded in 2018 Gross Domestic Bruto on Update Report Index Industrial Small and Middle Manufacturing showed GDP portion from Industrial Manufacture Non Oil is around 19\% and the trend show positively increased year on year 2015- 2018. Highly contribution from Small and Middle Industrial higly correlated from the changes and advance technology. Therefore, GAIKINDO, Indonesia Joint Industrial Motor Vehicle, forecast the sales of motor vehicle in Indonesia will continue increasingly trend positive. In the mean time Indonesia Motor Vehicle Industrial Association recorded the fluctuation result from distribution and production motor vehicle for period of lately ten years ago.

The situation in uncertainty economy recently, it is more difficult for small and middle business, this study use example of spare part producer of Under Bracket Motor vehicle, this. PT Karya Komponen Presisi as manufacturer of automotive parts in Cikarang, West Java, Indonesia, starting in 2015 in machineries and. The company wants to create a new project to a new type of bracket for the next five years start in 2019 with affordable priceas IDR 22.610/ pcs and offering discount around 0,5\% - 10\%. Previous project which is categorize as similar typical project with this Bracket $\mathrm{A}$, the company experience loss after decreasing demand of 
domestic automotive market. After a previous loss experience, management of PT Karya Komponen Presisi decide wants to do a financial feasibility study carefully. To support its mission as manufacturing company in the field of metal cutting for producing good quality automotive parts at competitive price.

\section{Conceptual Framework}

Feasibility Study, Kasmir and Jakfar (2012) explained that business feasibility study is an in depth analysis to study about business or investment whether feasible to run or not, equiped by quantitative and qualitative method and consider several aspects such as from market perspective, technical, financial, management, production, human resources and environmental aspect as recommendation tools.

Mixed methods with quantitative data from PDB forecast, inflation Central Bank, historic time series data from relevant project such as investment costs, forecast project calculation, operational cost and production cost. In addition, qualitative data including assumption scenario sensitivity analysis, five porter forces and business risks. The data study conducting in PT Karya Komponen Presisi from Mei 2018 until September 2018.

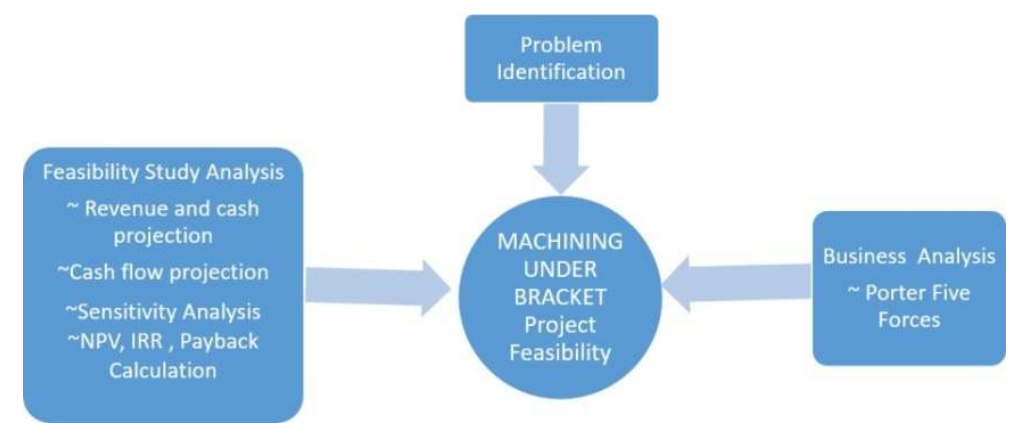

Fig.1. Conceptual Framework

\section{Result and Discussion}

The Range of selling price has minimum price with $10 \%$ discount as Rp $20.349 /$ pcs , maximum price with $0 \%$ discount $\mathrm{Rp} 22.610 / \mathrm{pcs}$, and middle range discount $5 \%$ as $\mathrm{Rp}$ 21.480 assume as normal price.

\subsection{Profit and Loss Forecast}

Profit and loss forecast in 2019 - 2023 show positive trend year on year. Total nett profit after tax from machining bracket project is IDR 13.594.180.460 at normal scenario production. Profit and Loss forecast will use to assume as cash in flow for the next year.

\subsection{Cash Flow Forecast}

Cashflow forecast in and out flow from three activities : Operating activity, Investing 
activities and Financing activities. Cashflow forecast found decreased trend from the first year of the new project until the fourth year because of fixed income. On the fifth year of the cashflow forecast, will have increased trend due to additional income assumption from salvage value as amount IDR 3.920.184.495.

This study used indirect method for cash flow forecast, the total cumulative statements of cash flow in the fifth year reached as IDR 14.398.370.285.

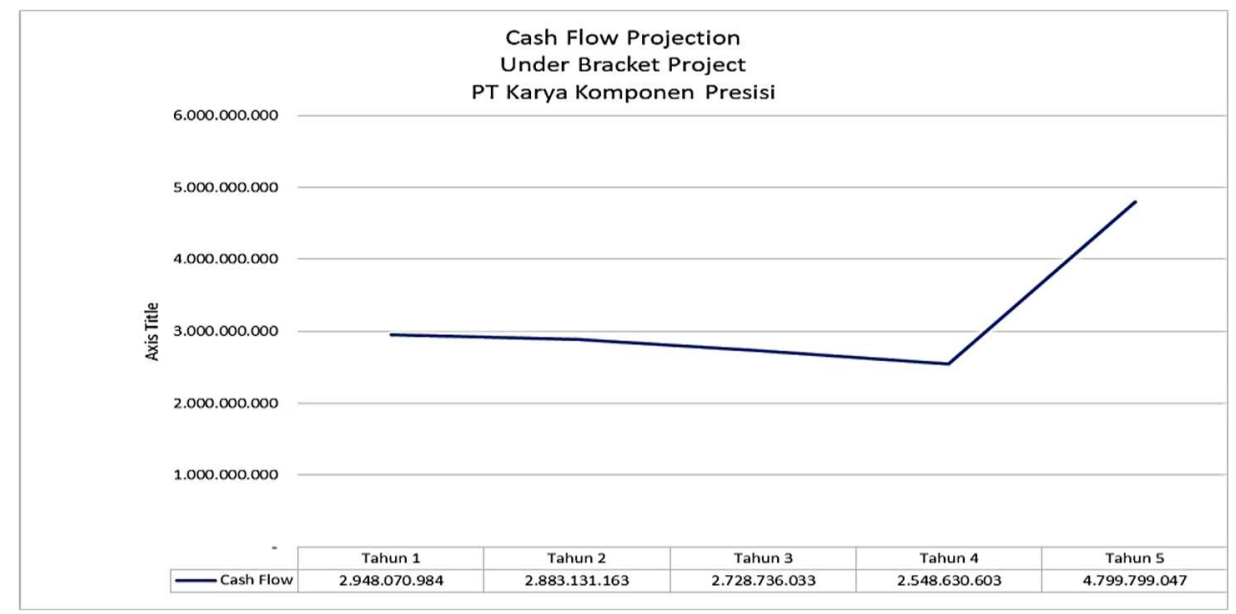

Fig.2. Cashflow Projection

Table 1. Cash flow projection

Cash Flow Projection (By Price Rp 21.480) PT Karya Komponen Presisi

\begin{tabular}{|c|c|c|c|c|c|c|}
\hline \multirow[t]{6}{*}{$\begin{array}{l}\text { Cash in Flow } \\
\text { (CIF) }\end{array}$} & & 2019 & 2020 & 2021 & 2022 & 2023 \\
\hline & Earning After Tax & 2.407 .454 .475 & 2.331 .054 .685 & 2.149 .413 .356 & 1.937 .524 .614 & 4.768 .733 .328 \\
\hline & Machine Depreciation & 444.376 .209 & 444.376 .209 & 444.376 .209 & 444.376 .209 & 444.376 .209 \\
\hline & Jig Depreciation & 119.250 .659 & 119.250 .659 & 119.250 .659 & 119.250 .659 & \\
\hline & $\begin{array}{l}\text { Prod Equipment } \\
\text { Depreciation }\end{array}$ & 31.821 .596 & 31.821 .596 & 31.821 .596 & 31.821 .596 & . \\
\hline & $\begin{array}{l}\text { Office Equipment } \\
\text { Depreciation }\end{array}$ & 4.286 .708 & 4.286 .708 & 4.286 .708 & 4.286 .708 & \\
\hline Total Cash in Flow & & 3.007 .189 .647 & 2.930 .789 .857 & 2.749 .148 .527 & 2.537 .259 .786 & 5.213 .109 .537 \\
\hline \multirow[t]{2}{*}{ Cash out Flow } & Lease Payable & & - & - & - & - \\
\hline & Deviden Payout & $361.118 .171,30$ & $349.658 .202,82$ & $322.412 .003,40$ & $290.628 .692,17$ & $715.309 .999,27$ \\
\hline \multicolumn{2}{|l|}{ Total Cash out Flow } & 361.118 .171 & 349.658 .203 & 322.412 .003 & 290.628 .692 & 715.309.999 \\
\hline \multicolumn{2}{|l|}{ Net Cash Flow } & 2.646 .071 .475 & 2.581 .131 .654 & 2.426 .736 .524 & 2.246 .631 .094 & 4.497 .799 .538 \\
\hline \multicolumn{2}{|l|}{ Beginning cash } & . & 2.646 .071 .475 & 5.227 .203 .129 & 7.653 .939 .653 & 9.900 .570 .747 \\
\hline \multicolumn{2}{|l|}{ Cummulative Cash } & 2.646 .071 .475 & 5.227.203.129 & 7.653.939.653 & 9.900 .570 .747 & 14.398 .370 .284 \\
\hline
\end{tabular}




\subsection{Investment Criteria Analysis}

\section{a) Net Present Value (NPV)}

Present value of cash flows minus initial investments. Discount rate assume as targeted profit company from the new project as $15 \%$. NPV value from the Machining Under Bracket project show positive value means this project feasible. The table below for five year calculate NPV as amount IDR 1.272.346.624.

Table 2. NPV calculation

\begin{tabular}{cccc}
\hline Year & Cash Flow & Disc Factor 15\% & PV 15\% \\
\hline Year 0 & $(8.096 .630 .014)$ & 0,869565217 & 2.300 .931 .718 \\
Year 1 & 2.646 .071 .475 & 0,756143667 & 1.951 .706 .355 \\
Year 2 & 2.581 .131 .654 & 0,657516232 & 1.595 .618 .656 \\
Year 3 & 2.426 .736 .524 & 0,571753246 & 1.284 .518 .619 \\
Year 4 & 2.246 .631 .094 & 0,497176735 & 2.236 .201 .290 \\
Year 5 & 4.497 .799 .538 & Total PV & 9.368 .976 .638 \\
& & Cash Investment & 8.096 .630 .014 \\
& & NPV & 1.272 .346 .624 \\
\hline
\end{tabular}

\section{b) Internal Rate of Return (IRR)}

Discount rate at which NPV $=0$ or PV inflows $=$ PV investment costs. Profitability index (PI)

Table 3. IRR \& PI Calculation

\begin{tabular}{cc}
\hline & Cash Flow \\
\hline Year 0 & $(8.096 .630 .014)$ \\
Year 1 & 2.646 .071 .475 \\
Year 2 & 2.581 .131 .654 \\
Year 3 & 2.426 .736 .524 \\
Year 4 & 2.246 .631 .094 \\
Year 5 & 4.497 .799 .538 \\
Discount rate & $15 \%$ \\
NPV & 1.272 .346 .624 \\
IRR & $21,0275 \%$ \\
PI & 1,1571 \\
\hline
\end{tabular}

c) Payback Period (PP)

$\mathrm{PP}=3+\frac{R p .442 .690 .361}{R p .2 .246 .631 .094}$
$\mathrm{PP}=3$ Year 3 Months

Table 4. Payback Period

\begin{tabular}{ccc}
\hline Year & Cash Flow & CF Cumulative \\
\hline Year 0 & $(8.096 .630 .014)$ & 8.096 .630 .014 \\
Year 1 & 2.646 .071 .475 & 5.450 .558 .539 \\
Year 2 & 2.581 .131 .654 & 2.869 .426 .885 \\
Year 3 & 2.426 .736 .524 & 442.690 .361 \\
Year 4 & 2.246 .631 .094 & 1.830 .940 .733 \\
Year 5 & 4.496 .799 .538 & 6.301 .740 .270 \\
\hline
\end{tabular}




\subsection{Sensitivity Analysis}

Table 5. Sensitivity Analysis

\begin{tabular}{llll}
\hline \multicolumn{1}{c}{ Indicator } & \multicolumn{3}{c}{ Scenario } \\
\cline { 2 - 4 } & \multicolumn{1}{c}{ Optimistic (133\%) } & \multicolumn{1}{c}{ Normal (100\%) } & \multicolumn{1}{c}{ Pessimistic (80\%) } \\
\hline Price & Rp 22.610 & Rp 21.480 & Rp 20.349 \\
Total production/Year & 576.000 & 432.000 & 345.600 \\
Sales & $\operatorname{Rp~} 65.116 .800 .000$ & Rp 46.395.720.000 & Rp 35.163.072.000 \\
Total Cost & Rp 43.506.481.816 & Rp 32.905.531.933 & Rp 27.076.230.891 \\
Net Cash Flow & Rp 21.076.300.248 & Rp 14.398.370.284 & Rp 10.899.909.186 \\
NPV & Rp 3.545.207.631 & Rp 1.272.346.624 & Rp (1.091.819.842) \\
IRR & $28,61 \%$ & $21,48 \%$ & $8,39 \%$ \\
Profitability Index & 1,34 & 1,16 & 0,87 \\
PP & 2 Year 8 Months & 3 Year 3 Months & 4 Year 2 Months \\
Remarks & Feasible & Feasible & Not Feasible \\
\hline
\end{tabular}

\subsection{Decision Tree}

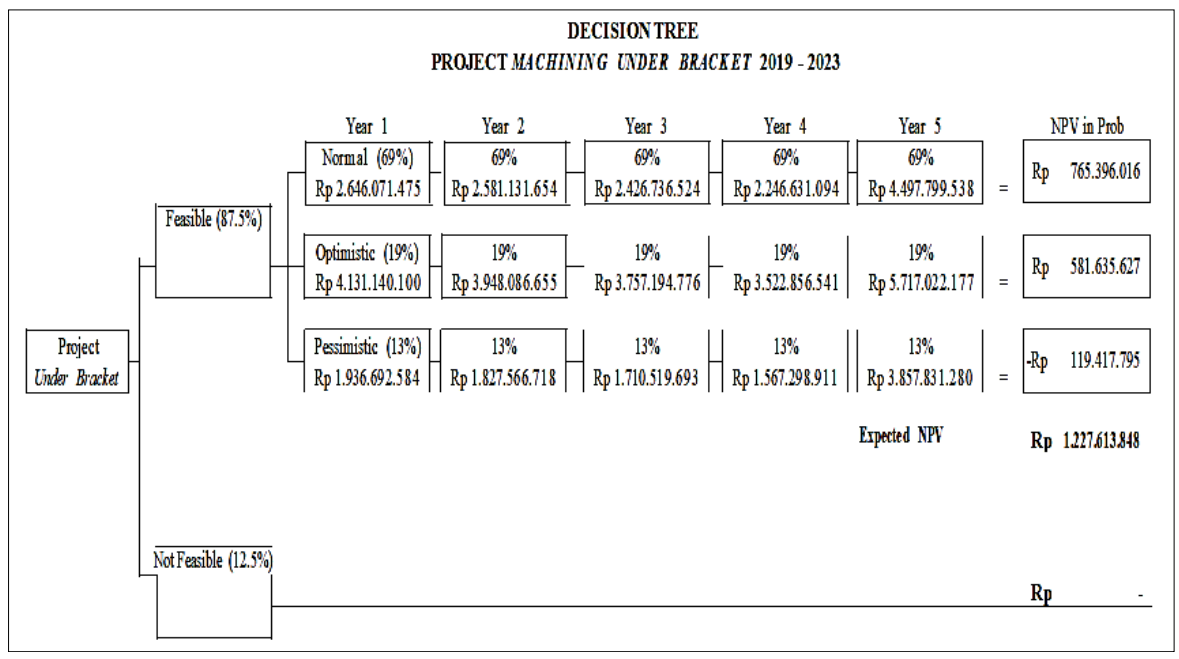

Fig.3. Decision Tree 


\subsection{Five Porter's Forces Analysis}

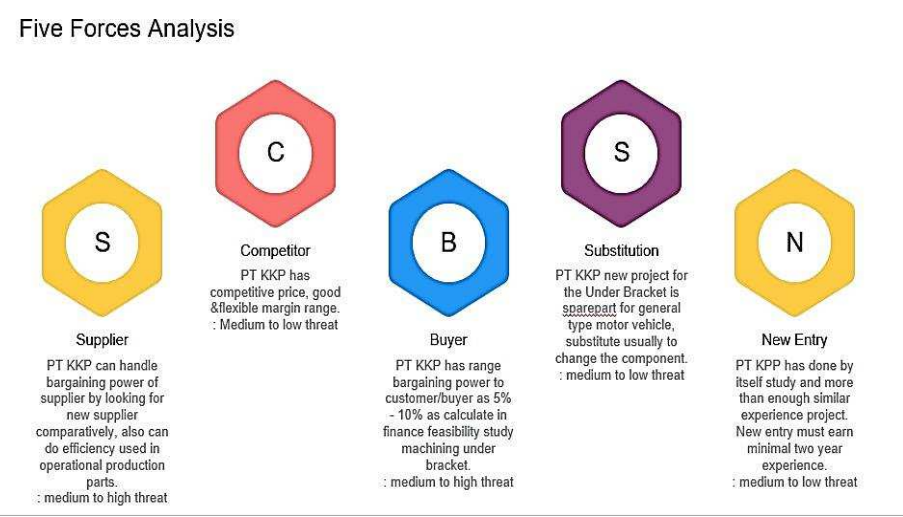

Fig.4. Five Forces Analysis

\subsection{Risk Analysis}

Overall probability total production volume fluctuated, with quantitative method for new project Machining Under Bracket has Standard Deviation as Rp 3.309.736.418. Distribution of risk cashflow shown as Coeffiecient of Variance 19.97\%. For qualitative method to scale risk analysis by summary the sources of risk internal and external company, the results shown in table Matrix Risk Analysis.

Table 6. Matrix Risk Analysis

\begin{tabular}{|c|c|c|c|c|c|c|}
\hline Scenario & Prob & Return & Expected Return & CF - E (R) & $(C F-E V)^{\wedge} 2$ & $\mathbf{p}(\mathbf{R}-\mathbf{E}(\mathbf{R}))^{\wedge} \mathbf{2}$ \\
\hline Normal & 0,688 & 14.398 .370 .284 & 9.898 .879 .571 & $-\operatorname{Rp} 814.804 .231$ & 663.905 .934 .738 .983 .000 & $456.435 .330 .133 .051 .000,00$ \\
\hline Optimistic & 0,188 & 21.076 .300 .248 & 3.951 .806 .297 & Rp 5.863.125.733 & Rp 34.376.243.362.072.500.000 & $\operatorname{Rp} 6.445 .545 .630 .388 .580 .000,00$ \\
\hline \multirow[t]{5}{*}{ Pessimistic } & 0,125 & 10.899.909.186 & 1.362 .488 .648 & -Rp 4.313.265.330 & Rp 18.604.257.802.965.900.000 & Rp 2.325.532.225.370.740.000,00 \\
\hline & & Expected Return & Rp 15.213.174.515 & & Variance & $\operatorname{Rp} 9.227 .513 .185 .892 .380 .000,00$ \\
\hline & & Std Deviasi & Rp 3.037.682.206 & & & \\
\hline & & Coeffiecient of & 0,199674447 & & & \\
\hline & & Var & $19,97 \%$ & & & \\
\hline
\end{tabular}

Table 7. Matrix Risk Analysis

\begin{tabular}{cc}
\hline No & Risk \\
\hline 1 & Production line stopped due to out of power PLN \\
2 & Production line stopped due to broken machineries \\
3 & Production line stopped Employees on strike \\
4 & Price consumable parts increased \\
5 & Total production volume fluctuated due to domestic \\
6 & market \\
\hline
\end{tabular}

\begin{tabular}{|c|c|c|c|c|c|}
\hline Probability & \multicolumn{5}{|c|}{ Effect } \\
\hline E (very often) & & & & & \\
\hline $\mathrm{D}$ (often) & & & & & \\
\hline $\mathrm{C}$ (moderate) & & & & & \\
\hline B (rarely) & & & $2 \& 4$ & & \\
\hline A (very rarely) & & 1 & 3 & & 6 \\
\hline
\end{tabular}

Diagonal colour from bottom left to up right : Low risk $\rightarrow$ Medium $\rightarrow$ High Risk $\rightarrow$ Extreme 


\section{References}

[1] Adiputra, M. R., \& Sukarno, S. Investment Feasibility for Iron Ore Mining Project. The Indonesian Journal of Business Administration ,2(4):pp 1695-1705, 2013.

[2] Adriano, W., \& Anggono, A. H. Feasibility Study and Investment Analysis - Textile Machineries at PT Heksatex Bandung. The Indonesian Journal of Business Administration , 2(8):pp 909-920 Motaz, 2013.

[3] Asosiasi Sepeda Motor Indonesia, from http://www.aisi.or.id/statistic/ 2018, Juni 21. Retrieved July 20, 2018

[4] BPS. Perkembangan Indeks Produksi Industri Manufaktur 2015-2017. Jakarta: Badan Pusat Statistik, 2017.

[5] Damayanti, Muhammad, A. R., \& Maya, S. Financial Feasibility Analysis of Gumanti Micro Hydro Power Plant Project. Journal of Business And Management, 4(3):pp 352-362, 2015.

[6] Dhote, D., \& Maroo, P. Feasibility Study of Capital Budgeting on Aluminium Casting Plant (Start Up). GE-International Journal of Management Research , 4 (4), pp 2394-4226, 2016.

[7] FME, T. Porter's FIve Forces Strategy Skills. Retrieved August 20, 2018, from http://www.freemanagement-ebooks.com/dldebk-pdf/fme-five-forces-framework.pdf, 2013.

[8] Hartono, S., Suryantini, A., \& Jamhari. Financial . Economic , And Environmental Feasibility Analysis of Palm Sugar Domestic Industry in Kolaka Indonesa. International Journal of Economics and Management Systems, Vol 1, 2016.

[9] Indonesia Investment. 2017, Juli 12., from https://www.indonesiainvestments.com:https://www.indonesia-investments.com/id/bisnis/industrisektor/otomotif/item6047?, Retrieved Mei 04, 2018.

[10] Karanovic, G., \& Suzana Baresa, S. B.2010. Techniques for Managing Projects Risk in Capital Budgeting Process. UTMS Journal of Economics , 1(2):pp 55-66.

[11] Liu, J., Jin, F., Xie, Q., \& Skirmore, M. Improving Risk Assesment in Financial Feasibility of International Engineering Project : A Risk Driver Perspective. International Journal of Project Management , pp 204-211, 2017.

[12] Muhammad Kholil, R. A. Analisis Kelayakan Investasi Workshop Pembuatan Spare Parts Mesin Industri Dengan Menggunakan Metode Kriteria Investasi. Journal of Industrial Engineering \& Management System, 8(2), August 2015.

[13] Osman, H., Saptari, A., \& Ismail, A. Y. Use of Sensitivity Analysis a Decision Support System in Automotive Painting Technology Investment. Indian Journal of Science and Technology, 10(43), November 2017.

[14] Permana, Y., Saleh, A., \& Bakar, A. April 2014. Analisis Kelayakan Usaha Pembuatan Spare Parts Dudukan Mesin dan Transmisi Mobil di Bekasi. Jurnal Online Institut Teknologi Nasional , 2(2):pp 376-387.

[15] Riky, A., \& H.mustamu, R. Porter Five Forces Model Pada PT Ruci Gas. AGORA Vol. 2(2):pp, 2014.

[16] Karanovic,G., Basera,S., \& Bogdan,S. Techniques for Managing Projects Risk in Capital Budgeting Process. UTMS Journal of Economics, 1(2):pp 55-66, 2010.

[17] Rosmawati, Rianda, L., Bahari, \& Taridala, S. A. Financial Feasibility Analysis of Cocoa Farming Business in Southeast Sulawesi. IOSR Journal of Business and Management,19(11):pp 60-65, November 2017. 